\title{
SUPORTE BÁSICO DE VIDA NA ESCOLA: O RELATO DA EVIDÊNCIA
}

\author{
M. BONÉ1 ${ }^{1}$ M. J. LOUREIRO ${ }^{2}$, J. BONITO ${ }^{3}$ \\ Universidade de Aveiro ${ }^{1,2}$, Universidade de Évora ${ }^{3}$ \\ ORCID ID: http://orcid.org/0000-0002-5600-0363 ${ }^{3}$ \\ jbonito@uevora.pt ${ }^{3}$
}

Submetido 30/08/2019 - Aceito 24/09/2020

DOI: $10.15628 /$ holos.2020.8959

\section{RESUMO}

Em alguns países, a educação de crianças em suporte básico de vida (SBV) é obrigatória, enquanto em muitos outros existem apenas iniciativas locais e regionais. Este trabalho objetiva conhecer fatores facilitadores e barreiras à aprendizagem de SBV e competências reveladas por alunos sujeitos à aprendizagem e treino de SBV, em países europeus, com base na revisão da literatura disponível. Foram analisados 29 artigos. Pode concluir-se que: a) as crianças apresentam-se recetivas e entusiasmadas, tornando a aprendizagem eficaz, com benefícios sociais, podendo ser multiplicadoras do ensino; $b$ ) os professores treinados podem ensinar tão eficazmente quanto os profissionais de saúde; c) a idade ideal para o início do ensino de compressões torácicas é cerca dos 12 anos; d) a proporção de espetadores treinados aumentará com a educação escolar.

PALAVRAS-CHAVE: suporte básico de vida, alunos, espetador, competências, empoderamento.

\section{BASIC LIFE SUPPORT IN SCHOOL: THE EVIDENCE REPORT}

\begin{abstract}
In some countries, basic life support (BLS) for children education is mandatory, while in many countries there are only local and regional initiatives. The objective of this work is to know the competences revealed by students subject to BLS learning in European countries, based on a review of available literature. We analyzed 29 articles. It can be concluded that: $a$ ) the children are receptive and
\end{abstract}

enthusiastic, making learning effective, with social benefits, and can be multipliers of teaching; b) trained teachers can teach as effectively as health professionals; c) the ideal age for beginning the teaching of chest compressions is about 12 years old; d) the proportion of trained bystanders will increase with school education.

KEYWORDS: basic life support, students, bystander, skills, empowerment. 


\section{INTRODUÇÃO}

A parada cardiorrespiratória (PCR) é um acontecimento inesperado que se apresenta como uma das principais causas de morte em todo o mundo (SNS, 2017), a terceira em nações industrializadas (Böttiger et al., 2017) que pode ser evitada (Semeraro et al., 2016). A maioria dos pacientes vítima de parada cardiorrespiratória fora-do-hospital (PCRFH) não apresenta sintomas ou desenvolve sintomas inespecíficos antes do evento. A prevenção primária da PCR, que afete os fatores de risco (Conroy et al., 2003) em pessoas com risco moderado a elevado, é uma medida importante na redução da sua incidência.

O aumento da taxa de sobrevivência em PCRFH pode ser conseguido através duma sequência de elos, conhecidos por "cadeia de sobrevivência" (Peberdy et al., 2010): a) reconhecimento precoce da PCR e ativação dos serviços de emergência; $b$ ) início rápido de suporte básico de vida (SBV) por espetadores, com ênfase nas compressões torácicas (CT); c) rápida desfibrilhação; $d$ ) serviços médicos de emergência básicos e avançados; e e) suporte avançado de vida e cuidados pós-PCR.

O SBV realizado pelo espetador corresponde a um conjunto de medidas articuladas, cujo objetivo é manter a ventilação e a circulação até à chegada de meios que possibilitem reverter a PCR. O SBV compreende a avaliação inicial da vítima, a manutenção da permeabilidade da via aérea, a ventilação com ar expirado e a compressão do tórax. A aplicação de medidas de SBV pelo espetador aumenta a probabilidade de sobrevivência da vítima até três vezes, quando iniciadas nos primeiros minutos após a PCRFH, por forma a evitar que aconteça a morte biológica, por lesão irreversível dos órgãos vitais (Sasson et al., 2010). Com SBV realizado pelo espetador, enquanto aguarda desfibrilhação, a taxa de sobrevivência média é de $3 \%-4 \%$, atuando como ponte para a desfibrilhação, o que revela a importante associação entre o SBV e a sobrevida, independentemente do tempo de desfibrilhação (Waalewijn et al., 2002).

Na Dinamarca, entre 2001 e 2012, a taxa de SBV realizado por espetadores passou de valores inferiores a 20\% para valores superiores a 50\% (Hansen et al., 2017a). De acordo com Hansen et al. (2017b), o êxito apresentado decorre das iniciativas efetuadas em todo o país, incluindo a educação obrigatória em SBV em escolas primárias, desde 2005. Uma das 10 recomendações, considerada pela Global Resuscitation Alliance (2013) como a mais importante para melhorar a sobrevivência em caso de PCRFH, é o aumento do número de espetadores. Vários estudos recentes reforçam a importância do SBV praticado por espetadores como fator que contribuiu para a melhoria da taxa de sobrevivência em caso de PCRFH (Geri et al., 2017; Kragholm et al., 2017; Riddersholm et al., 2017; Semeraro et al., 2016; Tanaka et al., 2018). A intervenção do espetador está associada à redução do tempo de permanência hospitalar e à admissão ao serviço de cuidados intensivos, contribuindo para uma melhor recuperação dos sobreviventes a PCRFH (Riddersholm et al., 2017).

Os objetivos deste trabalho são conhecer fatores facilitadores e barreiras à aprendizagem de SBV e conhecer as competências em SBV reveladas por alunos sujeitos à sua aprendizagem e treino, em países europeus, com base na revisão da literatura disponível. 


\section{CAPACITAÇÃO DE ESPETADORES EM SUPORTE BÁSICO DE VIDA}

Desde 1989, o European Resuscitation Council (ERC) estabelece guidelines na Europa, para a prática de ressuscitação cardiorrespiratória (RCP) e para a formação. O objetivo é formar nações de salva-vidas, comunidades céleres e eficazes na resposta à PCRFH (ERC, 2018). O ERC indica como medidas a educação neste domínio, o treino regular e pessoas motivadas para a pesquisa e para a atuação (Perkins et al., 2018). O treino de crianças em idade escolar em SBV é atualmente endossado pela Organização Mundial de Saúde. Em alguns países, a educação escolar de crianças em SBV já é obrigatória e, em muitos outros, iniciativas locais, regionais e nacionais foram iniciadas. Presentemente, são cinco os países europeus onde o ensino do SBV, nas escolas, se encontra legislado: Bélgica, Dinamarca, França, Itália e Portugal (Böttiger et al., 2015).

As European Resuscitation Council Guidelines for Resuscitation 2017 (Perkins et al., 2018) revelam que iniciativas diversas de saúde pública incrementam o êxito nas taxas de SBV dos espetadores, reforçando a importância da sua capacitação. Em regra, a taxa de sobrevivência a uma PCRFH varia muito, dependendo do local onde se vive. Na década de 1990, Bonito (1999) já expunha e defendia o lema "uma pessoa um socorrista", por forma a acontecer uma globalização na prestação eficaz de ajuda a uma vítima de PCRFH.

A taxa de sobrevivência à PCRFH, em algumas comunidades, é próxima de zero, enquanto em outras é de mais de $50 \%$ para fibrilhação ventricular (Global Resuscitation Alliance, 2013). Para reduzir a disparidade registada, a Global Resuscitation Alliance (2013) aponta 10 ações facilitadoras: i) Estabelecer um registo de PCRFH; ii) Implementar orientações para SBV, a disponibilizar via telefone com treino e melhoria contínua da qualidade; iii) Implementar SBV de alto desempenho com treino e melhoria contínua da qualidade; iv) Implementar resposta rápida dos serviços de socorro; v) Registar todas as tentativas de SBV, através de gravações audio e de ecocardiograma; vi) Capacitar as forças policiais para o uso de desfibrilhador automático externo (DAE); vii) Implementar programas para uso de DAE em locais públicos; viii) Estabelecer parcerias para financiamento de treino e melhoria contínua da qualidade; $i x$ ) Instituir hipotermia em todos os hospitais para os pacientes, após a RCP, se dela beneficiarem; e $x$ ) Trabalhar no sentido da excelência.

$\mathrm{Na}$ lista de ações apresentada, destacamos o treino obrigatório de SBV e de DAE em escolas e comunidades. A escola constitui-se como uma instituição que tem a oportunidade de contribuir para o pleno desenvolvimento dos alunos, integrando as esferas da formação académica, da personalidade e da cidadania. Em idade escolar, as crianças e os jovens apresentam facilidade em adquirir competências, conhecimentos e atitudes. Por outro lado, os professores, especialistas em educação, desde que capacitados, podem ensinar sem dificuldade técnicas de SBV durante a escolaridade.

Recentemente, a British Heart Foundation (BHF) (2018) revela que Glasgow é a primeira autoridade local na Escócia a trabalhar com vista a garantir que cada aluno do ensino secundário (com idades compreendidas entre os 11 e os 16 anos) receba treino em SBV. Para além disso, é também desenvolvido trabalho com diretores de escolas e outros decisores locais em educação. Esta iniciativa tem sido aclamada como um movimento pioneiro e de salva-vidas pela BHF. A estratégia Save a Life for Scotland foi lançada em 2015 e está prevista a sua conclusão em 2020. 
Em Portugal, as metas curriculares da disciplina de Ciências Naturais, do 9 o ano de escolaridade, homologadas pelo Ministério da Educação e Ciência em 2014, introduzem, pela primeira vez no ensino básico, a aprendizagem obrigatória do SBV (Bonito, 2014), vindo a ser confirmadas pelas Aprendizagens Essenciais (DGE, 2018).

\section{METODOLOGIA}

Este estudo seguiu o processo de revisão da literatura, preconizado por Bryman (2012), estruturado em 4 etapas: a) definição do objetivo e do foco do trabalho a realizar; b) procura de estudos relevantes publicados; $c$ ) avaliação dos estudos selecionados; e $d$ ) síntese dos resultados. Partindo dos objetivos, foram consideradas as seguintes questões de investigação: Q1- Que fatores se apresentam como facilitadores da aprendizagem de SBV?; Q2 - Que fatores se apresentam como barreiras à aprendizagem de SBV?; Q3 - Que competências revelam as crianças e os jovens sujeitos à aprendizagem e ao treino de SBV, em países europeus?

Para a seleção do corpus foi consultada, entre julho e agosto de 2018, a produção científica disponível em bases de dados: MEDLINE, ScienceDirect, SciELO, Google Scholar e Scopus. As pesquisas realizaram-se recorrendo a palavras-chave: basic life support, teaching, training, students, bystander, skills associando-lhe o operador booleano and. Consideraram-se critérios de inclusão a temática dos estudos, que devia permitir dar resposta às questões de investigação; a data da publicação, compreendida entre 2007-2018; estudos realizados em países europeus; e a disponibilização do texto completo. Foram critérios de exclusão estudos sem peritagem e estudos realizados em países não europeus.

Contabilizaram-se 1678 estudos que foram reduzidos a 43 após a análise dos seus títulos, selecionando-se aqueles que continham as palavras-chave e que se referiam à temática em estudo. Foi realizada a sua leitura integral seguida do crivo de uma adaptação da checklist para avaliação de estudos quantitativos Health Care Practice R\&D Unit (HCPRDU) evaluation tool for quantitative studies (Long, et al., 2002), tendo-se apurado 29 estudos (E1-E29) para a constituição do corpus (Tabela 1). 
Tabela 1: Constituição do corpus dos estudos.

\begin{tabular}{|c|c|c|c|c|c|c|c|c|c|c|}
\hline \multirow[b]{2}{*}{ Caraterísticas } & \multicolumn{9}{|c|}{ ESTUDOS } & \multirow[b]{2}{*}{ E10 } \\
\hline & E1 & E2 & E3 & E4 & E5 & E6 & E7 & E8 & E9 & \\
\hline Autores & $\begin{array}{l}\text { Tavares, Pedro } \\
\text { e Urbano } \\
(2016)\end{array}$ & Gala (2014) & $\begin{array}{l}\text { Mpotos e } \\
\text { Iserbyt (2017) }\end{array}$ & $\begin{array}{l}\text { Iserbyt, Charlierb } \\
\text { e Molsa (2014) }\end{array}$ & $\begin{array}{l}\text { De Buck et al. } \\
\text { (2015) }\end{array}$ & $\begin{array}{l}\text { Iserbyt e Byra } \\
\text { (2013) }\end{array}$ & $\begin{array}{l}\text { Semeraro et al. } \\
\text { (2017) }\end{array}$ & $\begin{array}{l}\text { Finke et al. } \\
\text { (2018) }\end{array}$ & $\begin{array}{l}\text { Meissner, } \\
\text { Kloppe e } \\
\text { Hanefeld (2012) }\end{array}$ & $\begin{array}{l}\text { Bollig, Wahl e } \\
\text { Svendsen (2009) }\end{array}$ \\
\hline País & Portugal & Portugal & Bélgica & Bélgica & Bélgica & Bélgica & Itália & Alemanha & Alemanha & Noruega \\
\hline Tipo de estudo & Revisão & Avaliação & Avaliação & Experimental & Revisão & Experimental & Longitudinal & Avaliação & Avaliação & Experimental \\
\hline Objetivos & $\begin{array}{l}\text { Conhecer a } \\
\text { idade a partir } \\
\text { da qual se deve } \\
\text { iniciar a } \\
\text { formação em } \\
\text { SBV. }\end{array}$ & $\begin{array}{l}\text { Avaliar os } \\
\text { conhecimentos } \\
\text { em SBV; } \\
\text { determinar a } \\
\text { relação de um } \\
\text { programa de } \\
\text { formação e a } \\
\text { aquisição da } \\
\text { competência } \\
\text { para realizar } \\
\text { SBV; identificar } \\
\text { fatores que } \\
\text { influenciam a } \\
\text { aquisição da } \\
\text { competência. }\end{array}$ & $\begin{array}{l}\text { Conhecer a } \\
\text { variabilidade na } \\
\text { profundidade de } \\
\text { compressão } \\
\text { para } 3 \text { grupos } \\
\text { etários (12-14; } \\
\text { 14-16; 16-18) } \\
\text { em função das } \\
\text { caraterísticas } \\
\text { físicas. }\end{array}$ & $\begin{array}{l}\text { Investigar se a } \\
\text { aprendizagem de } \\
\text { SBV a partir do } \\
\text { vídeo. }\end{array}$ & $\begin{array}{l}\text { Desenvolver um } \\
\text { caminho } \\
\text { educacional } \\
\text { baseado em } \\
\text { evidências para } \\
\text { permitir a } \\
\text { integração de } \\
\text { primeiros } \\
\text { socorros no } \\
\text { currículo escolar. }\end{array}$ & $\begin{array}{l}\text { Conhecer a } \\
\text { importância do } \\
\text { design das } \\
\text { ferramentas } \\
\text { usadas. }\end{array}$ & $\begin{array}{l}\text { Avaliar o uso do } \\
\text { jogo "Relive" em } \\
\text { alunos. }\end{array}$ & $\begin{array}{l}\text { Conhecer } \\
\text { aspetos do } \\
\text { desempenho } \\
\text { prático e teórico } \\
\text { de SBV da } \\
\text { literatura atual. }\end{array}$ & $\begin{array}{l}\text { Avaliar o } \\
\text { conhecimento } \\
\text { do SBV e o } \\
\text { desempenho de } \\
\text { alunos do } \\
\text { ensino médio } \\
\text { antes e depois } \\
\text { do treino. }\end{array}$ & $\begin{array}{l}\text { Determinar se um } \\
\text { programa de } \\
\text { ensino de } \\
\text { primeiros socorros } \\
\text { e de treino teórico } \\
\text { e prático pode } \\
\text { influenciar o seu } \\
\text { desempenho } \\
\text { numa situação de } \\
\text { primeiros } \\
\text { socorros. }\end{array}$ \\
\hline Instrumentos & & Questionário & $\begin{array}{l}\text { Grelha de } \\
\text { observação }\end{array}$ & $\begin{array}{l}\text { Grelha de } \\
\text { observação }\end{array}$ & & $\begin{array}{l}\text { Grelha de } \\
\text { observação }\end{array}$ & $\begin{array}{l}\text { Grelha de } \\
\text { observação }\end{array}$ & & $\begin{array}{l}\text { Grelha de } \\
\text { observação }\end{array}$ & $\begin{array}{l}\text { Grelha de } \\
\text { observação }\end{array}$ \\
\hline Sujeitos & & $\begin{array}{l}\text { Alunos }(\mathrm{N}=234) \text { : } \\
9 \text { o ano }(\mathrm{N}=118) \\
\text { e } \\
12^{\circ}(\mathrm{N}=116) .\end{array}$ & $\begin{array}{l}\text { Alunos }(\mathrm{N}=265) \text { : } \\
\text { masculino } \\
(\mathrm{N}=154) \text { e } \\
\text { feminino } \\
(\mathrm{N}=111)\end{array}$ & $\begin{array}{l}\text { Alunos ( } \mathrm{N}=128) \text {. } \\
\text { Idade média: } 17 \\
\text { anos. }\end{array}$ & $\begin{array}{l}\text { Estudos sobre } \\
\text { educação em } \\
\text { primeiros } \\
\text { socorros para } \\
\text { alunos até } 18 \\
\text { anos. }\end{array}$ & $\begin{array}{l}\text { Alunos ( } \mathrm{N}=111) \text {. } \\
\text { Idade média: } 13 \\
\text { anos. }\end{array}$ & $\begin{array}{l}\text { Alunos }(\mathrm{N}=65) \text {. } \\
\text { Idades: } 11-14 \\
\text { anos. }\end{array}$ & & $\begin{array}{l}\text { Alunos ( } \mathrm{N}=132 \text { ). } \\
\text { Idade média: } \\
14,6 \text { anos. }\end{array}$ & $\begin{array}{l}\text { Alunos ( } \mathrm{N}=228) \\
\text { Idades:5-7 anos. }\end{array}$ \\
\hline HOLOS, Ano & 36, v.6, e895 & 2020 & & & & 5 & & & & \\
\hline
\end{tabular}




\begin{tabular}{|c|c|c|c|c|c|c|c|c|c|c|}
\hline \multirow[b]{2}{*}{ Caraterísticas } & \multicolumn{10}{|c|}{ ESTUDOS } \\
\hline & E11 & E12 & E13 & E14 & E15 & E16 & E17 & E18 & E19 & E20 \\
\hline Autores & $\begin{array}{l}\text { Bollig, } \\
\text { Myklebust e } \\
\varnothing \text { stringen } \\
\text { (2011) }\end{array}$ & $\begin{array}{l}\text { Lorem, Palm e } \\
\text { Wikbc (2008). }\end{array}$ & $\begin{array}{l}\text { Kanstad, Nilsena } \\
\text { e Fredriksenb } \\
\text { (2011). }\end{array}$ & $\begin{array}{l}\text { Salciccioli et al. } \\
\text { (2017) }\end{array}$ & $\begin{array}{l}\text { Connoll et al. } \\
\text { (2007). }\end{array}$ & $\begin{array}{l}\text { Toner, Connolly } \\
\text { Laverty e } \\
\text { McGrath (2007) }\end{array}$ & Hill et al. (2009) & $\begin{array}{l}\text { Jones et al. } \\
\text { (2007) }\end{array}$ & $\begin{array}{l}\text { Malta Hansen et } \\
\text { al. (2017). }\end{array}$ & $\begin{array}{l}\text { Aaberg et al. } \\
\text { (2014) }\end{array}$ \\
\hline País & Noruega & Noruega & Noruega & Reino Unido & Reino Unido & Reino Unido & Reino Unido & Reino Unido & Dinamarca & Dinamarca \\
\hline Tipo de estudo & $\begin{array}{l}\text { Quasi- } \\
\text { experimental }\end{array}$ & $\begin{array}{l}\text { Quasi- } \\
\text { experimental }\end{array}$ & Avaliação & Avaliação & $\begin{array}{l}\text { Quasi- } \\
\text { experimental }\end{array}$ & $\begin{array}{l}\text { Quasi } \\
\text { experimental }\end{array}$ & $\begin{array}{l}\text { Quasi } \\
\text { experimental }\end{array}$ & $\begin{array}{l}\text { Observacional } \\
\text { descritivo }\end{array}$ & Avaliação & $\begin{array}{l}\text { Quasi } \\
\text { experimental }\end{array}$ \\
\hline Objetivos & $\begin{array}{l}\text { Avaliar os } \\
\text { efeitos de um } \\
\text { curso de } \\
\text { primeiros } \\
\text { socorros para } \\
\text { crianças de } 4 \text { a } 5 \\
\text { anos, dadas por } \\
\text { instrutor de } \\
\text { primeiros } \\
\text { socorros e } \\
\text { educadores. }\end{array}$ & $\begin{array}{l}\text { Conhecer o } \\
\text { número de } \\
\text { leigos } \\
\text { treinados } \\
\text { pelos kits } \\
\text { levados pelos } \\
\text { alunos para } \\
\text { casa e as } \\
\text { habilidades de } \\
\text { SBV } \\
\text { alcançadas. }\end{array}$ & $\begin{array}{l}\text { Conhecer } \\
\text { facilitadores e } \\
\text { barreiras à } \\
\text { atuação em } \\
\text { cenários realistas } \\
\text { por jovens } \\
\text { treinados em } \\
\text { SBV. }\end{array}$ & $\begin{array}{l}\text { Avaliar práticas } \\
\text { de treino em } \\
\text { SBV em escolas } \\
\text { secundárias de } \\
\text { Londres }\end{array}$ & $\begin{array}{l}\text { Avaliar o } \\
\text { conhecimento } \\
\text { retido em } \\
\text { SBV, } 6 \text { meses } \\
\text { após um } \\
\text { curso de } \\
\text { instrução para } \\
\text { crianças em } \\
\text { idade escolar. }\end{array}$ & $\begin{array}{l}\text { Avaliar a eficácia } \\
\text { de um programa } \\
\text { de instrução de } \\
\text { SBV com modelo } \\
\text { de treino de } 3 \\
\text { níveis: } \\
\text { estudantes de } \\
\text { medicina } \\
\text { ensinam os } \\
\text { professores, que } \\
\text { depois ensinam } \\
\text { os seus alunos. }\end{array}$ & $\begin{array}{l}\text { Avaliar a eficácia } \\
\text { com que os } \\
\text { alunos podem } \\
\text { realizar SBV e } \\
\text { estabelecer se o } \\
\text { desempenho é } \\
\text { afetado pela } \\
\text { relação entre as } \\
\text { CT e as } \\
\text { ventilações. }\end{array}$ & $\begin{array}{l}\text { Determinar em } \\
\text { que idade as } \\
\text { crianças podem } \\
\text { realizar CT } \\
\text { eficazes em } \\
\text { SBV. }\end{array}$ & $\begin{array}{l}\text { Avaliar o treino } \\
\text { em SBV dos } \\
\text { alunos após } 8 \\
\text { anos da sua } \\
\text { inclusão } \\
\text { legislativa. }\end{array}$ & $\begin{array}{l}\text { Avaliar o } \\
\text { conhecimento } \\
\text { sobre SBV em } \\
\text { estudantes do } \\
\text { ensino médio } \\
\text { dinamarqueses e } \\
\text { os benefícios de } \\
\text { uma única sessão } \\
\text { de treino por } \\
\text { médicos } \\
\text { juniores. }\end{array}$ \\
\hline Instrumentos & $\begin{array}{l}\text { Registo de } \\
\text { observação }\end{array}$ & $\begin{array}{l}\text { Teste de } \\
\text { habilidades }\end{array}$ & Questionário & $\begin{array}{l}\text { Questionário } \\
\text { Entrevista }\end{array}$ & $\begin{array}{l}\text { Grelha de } \\
\text { observação }\end{array}$ & Questionário & $\begin{array}{l}\text { Grelha de } \\
\text { observação }\end{array}$ & $\begin{array}{l}\text { Grelha de } \\
\text { observação }\end{array}$ & $\begin{array}{l}\text { Questionário e } \\
\text { entrevista }\end{array}$ & Questionário \\
\hline Sujeitos & $\begin{array}{l}\text { Alunos ( } \mathrm{N}=10 ; \\
50 \% \text { masculino). } \\
\text { Idades: } 4-5 \\
\text { anos. }\end{array}$ & $\begin{array}{l}\text { Alunos e } \\
\text { adultos } \\
(\mathrm{N}=200)\end{array}$ & $\begin{array}{l}\text { Alunos( } \mathrm{N}=376) \text {. } \\
\text { Idades: } 16-19 \\
\text { anos. }\end{array}$ & $\mathrm{N}=65$ escolas & $\begin{array}{l}\text { Alunos com } \\
\text { idades entre } \\
10-12 \text { anos. }\end{array}$ & $\begin{array}{l}\text { Alunos (10-12 } \\
\text { anos) e } \\
\text { professores. }\end{array}$ & $\begin{array}{l}\text { Alunos }(\mathrm{N}=85) \text {. } \\
\text { Idades: } 10-11 \\
\text { anos. }\end{array}$ & $\begin{array}{l}\text { Alunos }(\mathrm{N}=157) \\
\text { Três grupos por } \\
\text { ano escolar: } 9- \\
10,11-12 \text { e } 13- \\
14 \text { anos. }\end{array}$ & $\begin{array}{l}\text { Diretores de } \\
\text { escolas } \\
\text { dinamarquesas } \\
\text { (N=1240) e } \\
\text { professores } \\
\text { (N=1381). }\end{array}$ & $\begin{array}{l}\text { Alunos ( } \mathrm{N}=651) \\
\text { Idades: } 17-21\end{array}$ \\
\hline
\end{tabular}




\begin{tabular}{|c|c|c|c|c|c|c|c|c|c|}
\hline \multirow[b]{2}{*}{ Caraterísticas } & \multicolumn{9}{|c|}{ ESTUDOS } \\
\hline & E21 & E22 & E23 & E24 & E25 & E26 & E27 & E28 & E29 \\
\hline Autor & Isbye et al. (2007) & $\begin{array}{l}\text { Cuijpers et al. } \\
\text { (2016). }\end{array}$ & $\begin{array}{l}\text { Petrić et al. } \\
\text { (2013) }\end{array}$ & $\begin{array}{l}\text { Schroeder et al. } \\
\text { (2017) }\end{array}$ & $\begin{array}{l}\text { Lukas et al. } \\
\text { (2016) }\end{array}$ & Bohn et al. (2012). & $\begin{array}{l}\text { Thovar, } \\
\text { Castañeda, e } \\
\text { Benazco (2015) }\end{array}$ & Miró et al. (2012) & $\begin{array}{l}\text { Fonseca et al. } \\
\text { (2016) }\end{array}$ \\
\hline País & Dinamarca & Holanda & Croácia & Alemanha & Alemanha & Alemanha & Espanha & Espanha & Espanha \\
\hline Tipo de estudo & Avaliação & Avaliação & Avaliação & Revisão & Longitudinal & Longitudinal & $\begin{array}{l}\text { Quasi } \\
\text { experimental }\end{array}$ & Revisão & Experimental \\
\hline Objetivos & $\begin{array}{l}\text { Avaliar o efeito da } \\
\text { distribuição em } \\
\text { massa de } \\
\text { materiais } \\
\text { instrucionais de } \\
\text { SBV. }\end{array}$ & $\begin{array}{l}\text { Avaliar se os } \\
\text { estudantes de } \\
\text { medicina e } \\
\text { professores de } \\
\text { educação física } \\
\text { podem treinar em } \\
\text { SBV alunos de } \\
\text { ensino médio. }\end{array}$ & $\begin{array}{l}\text { Avaliar as } \\
\text { atitudes dos } \\
\text { alunos e dos seus } \\
\text { pais em treino de } \\
\text { SBV nas escolas e } \\
\text { as perceções } \\
\text { sobre medos dos } \\
\text { alunos em aplicar } \\
\text { e treinar SBV. }\end{array}$ & $\begin{array}{l}\text { Elucidar aspetos } \\
\text { educacionais do } \\
\text { ensino de crianças } \\
\text { em SBV e resumir } \\
\text { campanhas } \\
\text { abrangentes de } \\
\text { SBV em } \\
\text { bystanders em } \\
\text { todo o mundo. }\end{array}$ & $\begin{array}{l}\text { Comparar } \\
\text { professores e } \\
\text { médicos de } \\
\text { emergência } \\
\text { como } \\
\text { instrutores em } \\
\text { SBV. Perceber a } \\
\text { eficácia dos } \\
\text { treinos, } \\
\text { anualmente, } \\
\text { durante } 6 \text { anos } \\
\text { (1, } 3 \text { e } 6 \text { anos). }\end{array}$ & $\begin{array}{l}\text { Avaliar o } \\
\text { desempenho em } \\
\text { SBV de alunos após } \\
\text { diferentes tipos de } \\
\text { treino, efeitos da } \\
\text { frequência (anual e } \\
\text { bianual), idade } \\
\text { inicial (10 e } 13 \\
\text { anos) e facilitador } \\
\text { (médico de } \\
\text { emergência e } \\
\text { professor). }\end{array}$ & $\begin{array}{l}\text { Avaliar a eficácia } \\
\text { de um programa } \\
\text { de intervenção } \\
\text { educativo na } \\
\text { melhoria do grau } \\
\text { de informação e } \\
\text { conhecimentos } \\
\text { teóricos e práti- } \\
\text { cos em SBV. }\end{array}$ & $\begin{array}{l}\text { Analisar as } \\
\text { iniciativas em } \\
\text { Barcelona, no } \\
\text { desenvolvimento } \\
\text { do programa } \\
\text { PROCES, dirigido a } \\
\text { estudantes de } 15 \\
\text { e } 16 \text { anos durante } \\
10 \text { anos. }\end{array}$ & $\begin{array}{l}\text { Examinar a eficácia } \\
\text { de uma "canção de } \\
\text { ressuscitação } \\
\text { cardiopulmonar" } \\
\text { na melhoria das } \\
\text { habilidades básicas } \\
\text { de SBV. }\end{array}$ \\
\hline Instrumentos & Questionário & $\begin{array}{l}\text { Grelha de } \\
\text { observação }\end{array}$ & Questionário & & Questionário & Questionário & Questionário & & Questionário \\
\hline Sujeitos & $\begin{array}{l}\text { Alunos ( } \mathrm{N}=6947) \text {. } \\
\text { Idades: } 12-14 \\
\text { anos. }\end{array}$ & $\begin{array}{l}\text { Alunos }(\mathrm{N}=144) \\
\text { aleatoriamente } \\
\text { selecionados Idades: } \\
13-16 \text { anos. }\end{array}$ & $\begin{array}{l}\text { Alunos ( } \mathrm{N}=301) \\
\text { Idades: } 12-15 \\
\text { anos. Pais ( } \mathrm{N}= \\
361) . \text { Idades: } 29- \\
64 \text { anos. }\end{array}$ & & $\begin{array}{l}\text { Alunos }(\mathrm{N}=261) \text {. } \\
\text { Idades:10-13-16 } \\
\text { anos. }\end{array}$ & $\begin{array}{l}\text { Alunos ( } \mathrm{N}=433) \\
\text { Idades: } 12-13 \text { anos. }\end{array}$ & $\begin{array}{l}\text { Alunos }(\mathrm{N}=171) \\
\text { dos } 20,3^{\circ} \text { e } 40 \\
\text { anos do ensino } \\
\text { secundário } \\
\text { obrigatório e 10 } \\
\text { ano do ensino } \\
\text { secundário não } \\
\text { obrigatório. }\end{array}$ & & $\begin{array}{l}\text { Estudantes } \\
(\mathrm{N}=608) . \text { Idades: } \\
12-14 \text { anos. }\end{array}$ \\
\hline
\end{tabular}




\section{RESULTADOS E DISCUSSÕES}

Numa primeira análise, utilizou-se como indicador os tipos de participante nos estudos em apreciação, que são, principalmente alunos e professores. Na análise seguinte, os estudos foram submetidos a uma grelha com os indicadores: autor, país de realização do estudo, tipo de estudo, instrumentos de recolha de informação, objetivo do estudo, resultados e conclusões.

Os estudos considerados são do tipo avaliativo $\left(f_{i}=10\right)$, quasi experimental $\left(f_{i}=7\right)$, experimental $\left(f_{i}=4\right)$, revisão da literatura $\left(f_{i}=4\right)$, longitudinal $\left(f_{i}=3\right)$ e observacional $\left(f_{i}=1\right)$. A análise das conclusões dos estudos teve enfoque na verificação da efetividade das ações desenvolvidas no âmbito do ensino e treino do SBV, em contexto curricular e em iniciativas levadas a efeito em países europeus onde a temática não integra o currículo nacional.

\subsection{Competências em suporte básico de vida}

Os alunos foram um grupo preferencial, de fácil acesso e que se apresenta motivado para o treino e a aquisição competências em SBV (E1). As abordagens educativas adotadas nos trabalhos analisados contribuiram para ganhos em competências de SBV (E1 a E29) e permitiram a sua manutenção, nomeadamente após 2 meses (E17; E22), 4 meses (E9), 6 meses (E10; A25) e 8 meses (E29), em crianças com idades compreendidas entre 10-11, 13-16, 6-7, 10-12 e 12-14 anos, respetivamente. Um estudo longitudinal, sobre treinos anuais, revelou que após 6 anos as habilidades de SBV foram mantidas apesar da interrupção dos treinos durante três anos (E25).

Os alunos de 16-19 anos de idade revelaram conhecimento do número de telefone de emergência e 90\% tinham domínio teórico para socorrer uma pessoa adulta não responsiva (E13). Neste estudo, $83 \%$ dos participantes estavam dispostos a realizar SBV perante uma PCRFH. A investigação realizada com alunos, com uma média de 13 anos de idade, expôs desempenhos significativamente melhores no grupo experimental para volumes de ventilação e para a profundidade da compressão torácica. Na frequência das CT e no posicionamento correto da mão não se encontraram diferenças significativas (E6).

O empoderamento em SBV pareceu ser facilitado pela utilização de imagens combinadas com instruções escritas e pelo uso de vídeos com instruções (E4). O jogo Relive revelou-se uma ferramenta eficaz na aquisição de competências em SBV, entre alunos com idades entre os 11-14 anos (E7). Num estudo realizado com alunos com idades entre os 12-14 anos, no qual se avaliou a eficácia do treino com recurso à entoação de uma canção, encontraram-se diferenças significativas sobre o conhecimento de SBV pelos estudantes, nas CT, 8 meses pós-intervenção, entre o grupo de controlo e o grupo experimental (E29).

Estudantes do sexo feminino demonstraram: maior motivação para participar do treino de SBV e para responder à PCRFH; pontuar mais num questionário de conhecimentos de SBV; conhecer o número nacional de emergência; promover um maior efeito multiplicador na capacitação de familiares e amigos em SBV (E8). Os estudantes do sexo masculino mostraram maior confiança na proficiência em SBV e revelaram executar CT mais profundas (E8). Um nível de excelência em SBV 
de $25 \%$ foi atingido por alunos do sexo masculino com idades entre 12-14 anos e por alunas de 1416 anos, e pode ser gradualmente melhorado para $60 \%$ e $90 \%$, respetivamente, de acordo com a idade e o sexo. Tal meta pode exigir mais esforço e maior treino para crianças mais novas, especialmente as do sexo feminino, e provavelmente será mais facilmente alcançada por crianças com peso $>50 \mathrm{~kg}(\mathrm{E} 3)$.

As competências alcançadas em SBV tenderam a ser mantidas pelos alunos. Dois meses após o treino, 99\% dos estudantes ainda realizava CT de forma correta. Os alunos com idades entre os 10-11 anos ( $N=85$ ) conseguiram realizar SBV eficaz dois meses após uma sessão de treino com a duração de duas horas (E17).

Encontraram-se diferenças estatisticamente significativas entre participantes em programa de formação (alunos com idades compreendidas entre 6-7 anos) comparativamente aos que não recebem treino, nomeadamente, na avaliação correta do estado de consciência, da ventilação, do conhecimento do número europeu de emergência, do fornecimento das informações corretas na chamada de emergência, do conhecimento correto da posição lateral de segurança e da manipulação correta da via aérea. Após 6 meses, mostraram diferenças estatisticamente significativas para 5 dos 6 itens testados (E10). Crianças instruídas em SBV revelaram um aumento significativo no nível de conhecimentos após a sessão de treino. O seu nível de conhecimentos diminuiu ao longo de um período de 6 meses, embora tenha permanecido significativamente maior do que o de um grupo comparável de crianças que nunca haviam sido treinadas (E15).

Nem sempre os alunos conseguiram executar plenamente todas as competências do algoritmo de SBV, após treino de habilidades (E1). Fatores facilitadores do treino de SBV nas escolas tendem a ser a existência de um coordenador local de treino, professores com competências para realizar o treino e o fácil acesso ao equipamento de treino (E19). O treino de SBV parece contribuir para que os alunos aumentem a sua autoconfiança e revelem maior vontade de realizar SBV (E13). Após o treino, 99,2\% relatam que se sentem confiantes, sendo o valor de $26,9 \%$ antes do treino (E9).

A variável idade teve influência na eficácia da realização do SBV. Alunos com idades entre os 10-11 anos ( $N=85$ ) conseguiram realizar SBV de forma eficaz dois meses após uma única sessão de treino com a duração de duas horas (E17). Esta faixa etária conseguiu atingir maior profundidade de CT quando se utiliza o ratio 15:2 em vez de 30:2 (E17). Alunos com idades entre 9-10 anos ( $\mathrm{N}=$ 157) conseguiram realizar SBV dois meses após uma única sessão de treino, comprimindo o tórax do manequim até à profundidade recomendada nas guidelines. Cerca de $19 \%$ dos alunos com 1112 anos e 45\% dos com 13-14 anos atingiu uma profundidade de compressão adequada. Em ambos os grupos, a percentagem de CT com a profundidade recomendada pelas guidelines foi maior quando foi usado o ratio 15:2 (E18).

Entre os estudantes do ensino secundário $(\mathrm{N}=651)$ com idades compreendidas entre 17-21 anos, $63 \%$ participou anteriormente em treino de SBV ministrado por médicos juniores. Apenas $28 \%$ conseguiu identificar a ventilação normal. A maioria receou complicar o estado de saúde da vítima ou causar-lhe a morte (E28). 
Verificou-se uma melhoria significativa nos resultados do questionário aplicado depois do treino. A proporção de estudantes que se sentiu bem preparado para realizar SBV aumentou de $30 \%$ para $90 \%$ (E20). Professores treinaram alunos com idades entre os 12-14 anos que, posteriormente, treinaram amigos e familiares com o kit disponibilizado para o efeito, promovendo um efeito formativo multiplicador (E21). Os alunos com idades compreendidas entre os 13-16 anos, 8 semanas após a formação, treinados por estudantes de medicina e professores de educação física, mostraram competência suficiente em SBV (E22).

A autoconfiança cresceu nos grupos de treino (E26). A frequência da realização dos treinos e a ministração por médicos não contribuiu para um melhor desempenho dos alunos relativamente aos promovidos por professores (E26). A assimilação de conceitos em SBV foi mais evidente em alunos após os 15 anos de idade. Alunos mais novos apresentaram dificuldades físicas, sugerindose concentrar os cursos de formação e treino em SBV nos grupos com idades superiores a 15 anos (E23).

\subsection{Abordagens educativas}

Foram diversas as abordagens adotadas nos treinos de SBV, em contexto escolar, nos estudos analisados (Tabela 2). A inclusão da formação em SBV nos curricula escolares no final 1은 ciclo do ensino básico (6-10 anos de idade) é recomendável e exequível (E2).

Tabela 2: Abordagens educativas no ensino do SBV.

\begin{tabular}{l|c}
\multicolumn{1}{c|}{ Abordagem educativa } & Estudos \\
\hline - Treino em SBV e questionário. & E4 \\
- Aprendizagem de SBV através de imagens combinadas e de instruções & E6 \\
escritas (10 grupo de alunos) e através de vídeos com instruções no ecrã & (20 grupo). \\
- No grupo de controlo, foram colocadas instruções escritas nos cartões de \\
tarefa. No grupo experimental, foram colocadas instruções escritas perto \\
da parte correspondente da imagem nos cartões de tarefa, refletindo a \\
aplicação do princípio de contiguidade espacial. \\
- Treino com recurso ao jogo Relive. \\
- Curso de treino prático tutorial de SBV, com duas horas de duração. Os \\
alunos foram convidados a realizar o cenário de PCRFH antes do treino. \\
Posteriormente, participaram no mesmo cenário e preencheram um \\
questionário para autoavaliação da sua confiança pré e pós-treino. \\
- Curso de primeiros socorros, em 5 aulas (45 min cada) de treino teórico e \\
prático para crianças de 6-7 anos de idade. \\
- Curso de primeiros socorros para crianças de 4-5 anos de idade, dado por \\
um instrutor de primeiros socorros e por educadores de infância. \\
- Treino de SBV tendo professores como facilitadores. Os alunos levaram o \\
kit (um pequeno manequim) para casa e expuseram o maior número \\
possível de membros da família e amigos (adultos) a treino. \\
- Questionário para avaliar conhecimento teórico. \\
HOLOS, Ano 36, v.6, e8959, 2020
\end{tabular}


- Auditoria prospetiva do treino de SBV nas escolas secundárias de Londres, contactadas por e-mail e entrevista por telefone.

- Um curso de SBV - o programa ABC for Life - para ensinar crianças de 10-

E14 12 anos. Os estudantes de medicina ensinaram professores para estes ensinarem os alunos. Respondem a questionário antes e depois da instrução.

- Treino em SBV de $2 \mathrm{~h}$. Após 2 meses, os alunos foram randomizados em dois grupos e realizaram SBV em manequim: 3 min no ratio 30:2 seguido de 5 min em repouso e depois 3 min no ratio 15:2.

- Treino em manequim de acordo com o cartão de competência nível 3 do programa de treino.

- Treino em SBV e entrevista aos professores e diretores.

- Treino de 45 min de SBV, incluindo aspetos teóricos e práticos com manequins. Aplicação de questionário antes do treino e de acompanhamento uma semana depois.

- Uso de DVD de instrução de 24 min em SBV e uso de kit para treinar

E16 familiares e amigos. Questionário sobre os sujeitos que treinaram.

- Treino e avaliação após oito semanas num cenário de PCRFH simulada em manequim, usando software e gravações vídeo.

- Treino de SBV por professores treinados ou por médicos de emergência.

- Treino de SBV e aplicação de questionário antes e 10 dias após a formação.

- Treino SBV com recurso à canção. Aplicação de questionário antes e após um e oito meses.

Fonte: Elaboração própria

Os estudos analisados concluiram que existe vantagem na implementação da formação de SBV na escola. Um caminho educacional baseado em evidência, com metas de aprendizagem para cada faixa etária, pode ser usado na integração do treino em SBV nos curricula (E5). As vantagens dessa introdução traduziram-se em ganhos nomeadamente, na motivação para a realização de manobras de SBV em PCRFH presenciada (E1).

\subsection{Idade de iniciação}

A idade favorável para o início da capacitação das crianças em SBV, apresentada nos estudos, foi variável. Foram apontados 4-5 (E11), 6-7 (A10) e os 8-9 anos de idade (E1, E26). Um nível mínimo de excelência no desempenho em SBV foi atingido por rapazes de 12-14 anos e por raparigas de 1416 anos, relacionando-se o peso superior a $50 \mathrm{~kg}$ com os melhores resultados verificados (E3). A idade mínima de 12-13 anos resultou em desempenhos de SBV eficazes, similarmente aos de adultos: $a$ ) deteção correta de PCRFH; $b$ ) pedido de ajuda corretamente realizado; $c$ ) CT eficazes; e d) se aplicável, o início de uma adequada ventilação boca-a-boca (E24). A implementação mundial de um treino de duas horas de SBV por ano, em crianças a partir dos 12 anos de idade, foi recomendada pela declaração "Kids Save Lives" desde 2015 (E24). Em Portugal, um estudo comparativo entre alunos do 9 (com 14 anos de idade) e 12 anos (com 17 anos de idade) de escolaridade revelou que a implementação do SBV deve acontecer no 12 을 ano (E2). 


\subsection{Recursos}

Nos treinos de SBV foram usados diversos recursos, nomeadamente canções, vídeos, imagens, jogos e kits. Os vídeos e as imagens foram facilitadores da aprendizagem de SBV (E4). O design de ferramentas instrucionais (e.g., cartões de tarefas) contribuiram para a aprendizagem dos alunos (E6), sendo esta mais significativa quando as palavras e imagens correspondentes surgiram em associação (E6). O jogo Relive é uma ferramenta útil para disseminar o conhecimento sobre SBV e melhorar as habilidades em crianças em idade escolar. A distribuição de kit de SBV por alunos com 12 anos de idade permitiu que uma percentagem significativa da população se concentrasse no treino em SBV (E12). O Programa de Reanimación Cardiopulmonar Orientado a Centros de Enseñanza Secundária (PROCES), usado por médicos especialistas em emergências, revelou-se uma excelente ferramenta para disseminar o conhecimento em SBV entre os estudantes com 15-16 anos (E28). A componente musical no ensino de SBV aumentou a eficácia e a capacidade de recordar o algoritmo de SBV (E29). O treino em SBV pode ser disseminado numa população distribuindo manequins de RCP entre as crianças (E12).

\subsection{Sexo}

Alunos do sexo feminino pareceram estar mais motivados a participar em treinos de SBV, assumindo o papel de multiplicadores, abrangendo mais pessoas em treino prático. Os estudantes do sexo masculino conseguiram uma profundidade de CT mais eficiente (E8).

\subsection{Peso}

O peso dos alunos tendeu a influenciar os desempenhos em SBV, pois a profundidade da CT mostrou uma relação significativa com a idade, peso e altura das crianças. Crianças pequenas, que ainda não são fisicamente capazes de comprimir adequadamente o tórax, podem aprender os princípios da CT como crianças mais velhas (E18).

\subsection{Atores da capacitação}

Diversos atores forneceram inputs e treinaram os alunos em SBV. A eficácia do treino ministrado por estudantes de medicina ou por professores de educação física não foi inferior ao treino assegurado por um enfermeiro (E23), sugerindo que professores e estudantes de medicina possam ser recrutados para o treino em SBV nas escolas (E23). Professores, previamente treinados por estudantes de medicina, podem ensinar SBV a crianças de 10-12 anos de idade, usando o programa $A B C$ for Life (E21). Uma sessão de treino prático por médicos juniores parece ser eficaz para capacitar espetadores (E20). Os professores treinados foram capazes de capacitar os seus alunos (E11; E12; E16; E20; E21; E22; E25; E26).

\subsection{Facilitadores}


Alunos (12-15 anos) e pais (29-64 anos) apresentaram uma atitude positiva em relação ao treino de SBV nas escolas (E23). Os principais aspetos a considerar para iniciar e manter um programa de SBV em escolas tendem a ser: a) Elaborar um programa específico para a idade dos alunos; b) Adaptar o programa às caraterísticas educacionais; c) Complementar o programa com aspetos transversais das disciplinas do curso; d) Envolver diretamente o corpo docente na conceção, planeamento de implementação e nas aulas; e) Envolver a totalidade dos alunos; f) Fornecer todo o material necessário; $g$ ) Programar um número suficiente de horas para o programa ser integrado no ano escolar, indo além de uma sessão única (idealmente, entre 5 e 10 horas); $h$ ) Concentrar a formação num período não superior a duas semanas; e i) Obter compromissos das administrações educativas (E28).

\subsection{Barreiras}

Um estudo desenvolvido em 65 escolas do distrito de Londres concluiu que são baixas as taxas de formação de SBV nas escolas secundárias. Registaram-se 5 escolas (8\%) que forneceram programas universais de treino para estudantes e 31 escolas (48\%) ofereceram treino como parte de um programa extracurricular ou módulo escolhido. A maioria das escolas ( $N=40 ; 61 \%)$ não tinha um DAE disponível(E14): estava disponível em 18 escolas (28\%). Sete das escolas (11\%) desconheciam a quantidade de DAE de que dispunham. As razões mais comuns para não existir um programa universal de treino em SBV foram a exigência de tempo de aula adicional (28\%) e financiamento não disponível para o programa (28\%) (E14).

Apesar da legislação dinamarquesa determinar a obrigatoriedade do treino em SBV na escola, este não foi implementado com sucesso (E19). O conhecimento das principais áreas do SBV é reduzido entre os estudantes com idades compreendidas entre os 17-21 anos (E20).

Os jovens noruegueses estavam motivados a realizar SBV mas surgiram barreiras quando foram apresentados cenários mais detalhados e realistas de PCRFH. Para estes alunos, a realização de SBV será comprometida se a vítima é um membro da família (74\%), uma criança (67\%) ou um consumidor de substâncias psicoativas intravenosas (18\%) (E13). O recurso a um modelo de treino de adultos através de kits, que os alunos usam com os seus familiares e amigos, tendeu a revelar que as crianças de 12 anos são demasiado jovens para exercerem influência na motivação dos adultos para o treino de SBV (E12).

\subsection{Vantagens}

Fornecendo aos alunos treino de qualidade em SBV na escola, a geração seguinte pode fortalecer os dois primeiros elos da cadeia de sobrevivência em PCRFH (A13). Embora os estudos referenciados demonstrem que nem sempre os alunos conseguem executar plenamente as competências do algoritmo de SBV, eles são um grupo preferencial, de fácil acesso e motivado e desperto para a realização de manobras de SBV (E1). O treino precoce reduziu a ansiedade em cometer erros e aumentou a disposição dos participantes para ajudar, na sua capacidade altruísta (E26). Os cursos tenderam a duplicar a confiança dos alunos naquilo que aprenderam, que thes 
permite salvar vidas (E26). Um programa de treino concebido e ensinado como parte do currículo escolar terá um impacto significativo na saúde pública (A15). Devido à importância no conhecimento do SBV, apresenta-se necessária a implementação de cursos bem organizados na escola, anualmente e a reavaliação do conhecimento de forma cíclica (E27).

\section{CONCLUSÃO}

A Constituição da República Portuguesa (1976) estabelece que todos têm direito à proteção da saúde e o dever de a defender e promover (art. 64으, no 1). A missão da educação não se esgota na transmissão de conhecimento académico, mas antes num despertar de consciências que permite o desenvolvimento do aluno como cidadão interventivo e pró-ativo. A capacitação de alunos em SBV, com vista à atuação eficaz na sociedade civil, é promotora de altruísmo e de sentido cívico, contribuindo para o aumento da taxa de sobrevivência em situação de PCRFH. A escola tem a oportunidade de contribuir para o desenvolvimento holístico dos alunos como pessoas sociais. Por esta razão, a escola é um local privilegiado para a ensinar SBV, pois acolhe a totalidade de crianças entre os 6-18 anos de idade. Os professores, desde que capacitados, podem, sem dificuldade, ensinar SBV aos seus alunos.

As conclusões dos estudos analisados indicam explicitamente ganhos mensuráveis em competências em SBV pelos alunos treinados. A idade ideal para a abordagem e treino do SBV varia, nos estudos considerados, entre os 4-18 anos, sendo os 12 anos a idade mais fortemente apontada. Os recursos tendem a ter influência favorável nos ganhos e manutenção das aprendizagens em SBV. A profundidade de compressão parece relacionar-se com a idade, peso e altura das crianças. Os professores apresentam-se como os principais atores de capacitação dos alunos e estes revelam-se motivados e apresentam uma atitude positiva em relação ao treino de SBV nas escolas. A excassez de tempo, de financiamento e a falta de equipamento comprometem a capacitação em SBV. As aprendizagens teórico-práticas realizadas pelos alunos tendem a manter-se, sendo que o reforço periódico dessas aprendizagens se revela pertinente quer ao nível de competências adquiridas quer ao nível do reforço da confiança para a atuação dos alunos.

Parece ser necessário um investimento em investigação, na definição de conteúdos a abordar, nas estratégias, nos recursos, no financiamento e no contínuo feedback com vista ao melhoramento cíclico das práticas. Parecem ser estes os pilares para o sucesso e para o consequente aumento de espetadores capacitados em SBV fortalecendo-se a relação simbiótica entre a educação e a saúde, entre o aluno e o cidadão.

\section{AGRADECIMENTOS}

Para a realização deste trabalho, contou-se com o apoio do Centro de Investigação em Didática e Tecnologia na Formação de Formadores (UID/CED/00194/2013) da Universidade de Aveiro. 


\section{REFERÊNCIAS}

Aaberg, A., Larsen, C., Rasmussen, B., Hansen, C., \& Larsen, J. (2014). Basic life support knowledge, self-reported skills and fears in Danish high school students and effect of a single 45-min training session run by junior doctors; a prospective cohort study. Scandinavian Journal of Trauma, Resuscitation and Emergency Medicine, 22(24), 1-6. Recuperado de https://sjtrem.biomedcentral.com/track/pdf/10.1186/1757-7241-22-24.

Bohn, A., Van Aken, H., Möllhoff, T., Wienzek, H., Kimmeyer, P., Wilde, E., ... Weber, T. (2012). Teaching resuscitation in schools: annual tuition by trained teachers is effective starting at age 10. A four-year prospective cohort study. Resuscitation 83, 619-625. Recuperado de https://www.einlebenretten.de/docman/fachpublikationen/25-teaching-resuscitation-inschools-annual-tuition-by-trained-teachers-is-effective-starting-at-age-10-a-fouryearprospective-cohort-study/file.html.

Bollig, G., Wahl, H., \& Svendsen, M. (2009). Primary school children are able to perform basic lifesaving first aid measures. Resuscitation, 80(6), 689-692. Recuperado de https://www.resuscitationjournal.com/article/S0300-9572(09)00133-6/fulltext.

Bollig, G., Myklebust, A. G., \& Østringen, K. (2011). Effects of first aid training in the kindergarten-a pilot study. Scandinavian Journal of Trauma, Resuscitation and Emergency Medicine, 19(13), 27. Recuperado de https://sjtrem.biomedcentral.com/track/pdf/10.1186/1757-7241-19-13.

Bonito, J. (1999). Urgente: socorristas precisam-se. Objectivo: salvar pessoas que estão ao nosso lado. Local: aqui mesmo. In J. Precioso et al. (Orgs.), Educação para a saúde (pp. 325-349). Braga, Portugal: Departamento de Metodologias da Educação da Universidade do Minho. Recuperado de http://hdl.handle.net/10174/4867.

Bonito, J., Morgado, M., Silva, M., Figueira, D., Serrano, M., Mesquita, J., \& Rebelo, H. (2014). Metas curriculares. Ensino básico. Ciências naturais. 9 ano. Direção-Geral da Educação, Portugal. Recuperado https://www.dge.mec.pt/sites/default/files/ficheiros/metas curriculares ciencias naturais 9 ano $0 . p d f$

Böttiger B., \& Van Aken, H. (2015). Kids save lives - Training school children in cardiopulmonary resuscitation worldwide is now endorsed by the World Health Organization (WHO). Resuscitation 94, A5-A7. Recuperado de https://www.resuscitationjournal.com/article/S03009572(15)00315-9/fulltext.

Böttiger, B., Semeraro, F., Altemeyer, K-W., Breckwoldt, J., Kreimeier, U., Rücker, G., ... Wingen, S. (2017). Kids save lives. School children education in resuscitation for Europe and the world. European Journal of Anaesthesiology, 34, 792-796. Recuperado de http://www.rcppediatrica.org/wp-content/uploads/2017/11/KIDS-SAVE-LIVESB\%C3\%B6ttiger-et-al.-EJA-2017.pdf. 
British Heart Foundation (2018). Glasgow pledges to train all secondary pupils in life saving CPR. Recuperado de https://www.bhf.org.uk/what-we-do/news-from-the-bhf/newsarchive/2018/june/glasgow-pledges-to-train-all-secondary-pupils-in-life-saving-cpr.

Bryman, A. (2012). Social research methods. Oxford, England: Oxford University Press.

Connolly, M., Toner, P., Connoly, D., \& McCluskey D. R. (2007). The 'ABC for life' programme teaching basic life support in schools. Resuscitation, 72(2), 270-9. Recuperado de https://www.ncbi.nlm.nih.gov/pubmed/17134814.

Conroy, R. M., Pyörälä, K., Fitzgerald, A. P., Sans, S., Menotti, A., De Backer, G., ... Wilhelmsen, L. (2003). Estimation of ten-year risk of fatal cardiovascular disease in Europe: The SCORE project. European Heart Journal, 24(11), 987-1003. Recuperado de https://academic.oup.com/eurheartj/article/24/11/987/427645.

Portugal (1976). Constituição da República Portuguesa. VII Revisão Constitucional de 2005.

Cuijpers, P., Bookelman, G., Kicken, W., De Vries, W., \& Gorgels, A. (2016). Medical students and physical education students as CPR instructors: an appropriate solution to the CPR - instructor shortage in secondary schools? Netherlands Heart Journal, 24(7-8), 456-461. Recuperado de https://rd.springer.com/article/10.1007\%2Fs12471-016-0838-2.

De Buck, E., Van Remoortel, H., Dieltjensa, T., Verstraeten, H., Matthieu, C., Moens, O., \& Vandekerckhove, P. (2015). Evidence-based educational pathway for the integration of first aid training in school curricula. Resuscitation, 94, 8-22. Recuperado de https://www.sciencedirect.com/science/article/pii/S0300957215002531\#!.

DGE - Direção-Geral da Educação (2018). Aprendizagens essenciais. Articulação com o perfil dos alunos. 9o ano. 3o ciclo do Ensino básico. Ciências naturais. Direção-Geral da Educação, Portugal. Recuperado de https://www.dge.mec.pt/aprendizagens-essenciais-ensino-basico.

ERC - European Resuscitation Council (2018). Bringing resuscitation to the world. Recuperado de https://www.erc.edu/.

Finke, S. R., Schroeder, D. C., Ecker, H., Wingen, S., Hinkelbein, J., Wetsch, W. A., ... Böttiger, B.W. (2018). Gender aspects in cardiopulmonary resuscitation by schoolchildren: A systematic review. Resuscitation, 125, 70-78. Recuperado de https://www.ncbi.nlm.nih.gov/pubmed/29408490.

Fonseca, F., Valle, J., Canales, N., Andrade, M., Siggers, A., \& Lopera, E. (2016). Basic life support knowledge of secondary school students in cardiopulmonary resuscitation training using a song. International Journal of Medical Education, 7, 237-241. Recuperado de https://www.ncbi.nlm.nih.gov/pubmed/27442599.

Gala, C. (2014). Competência dos alunos em SBV: Estudo comparativo entre o 9o ano e o 12. o ano de escolaridade (Master's thesis, Instituto Politécnico de Viseu). Recuperado de http://repositorio.ipv.pt/handle/10400.19/2550. 
Geri, G., Fahrenbruch, C., Meischke, H., Weaver, M., White, L., Rea, T., \& Weaver, M. (2017). Effects of bystander CPR following out-of-hospital cardiac arrest on hospital costs and long-term survival. Resuscitation, 115, 129-134. Recuperado de https://www.resuscitationjournal.com/article/S0300-9572(17)30169-7/fulltext.

Global Resuscitation Alliance (2013). 10 Steps for improving survival from sudden cardiac arrest. Recuperado

http://www.globalresuscitationalliance.org/downloads/ebook/TenStepsforlmprovingSurvivalF romSuddenCardiacArrest-RA-eBook-PDFFinal-v1_2.pdf.

Hansen, C., Rosenkranz, S., Folke, F., Zinckernagel, L., Tjørnhøj-Thomsen, T., Scient, M. ... Rod, M. (2017a). Lay bystanders' perspectives on what facilitates cardiopulmonary resuscitation and use of automated external defibrillators in real cardiac arrests. Journal of the American Heart Association 6(3), e004572. Recuperado de https://www.ncbi.nlm.nih.gov/pmc/articles/PMC5524003/.

Hansen, C., Zinckernagel, L., Kjær Ersbøll, A., Tjørnhøj-Thomsen, A., Wissenberg, M., Lippert, F., ... Folke, F. (2017b). Cardiopulmonary resuscitation training in schools following eight years of mandating legislation in Denmark: a nationwide survey. Journal of the American Heart Association, 6(3), e004128. Recuperado de http://jaha.ahajournals.org/content/6/3/e004128.full.

Hill, K., Mohan, C., Stevenson, M., \& McCluskey, D. (2009). Objective assessment of cardiopulmonary resuscitation skills of 10-11-year-old schoolchildren using two different external chest compression to ventilation ratios. Resuscitation, 80(1), 96-9. Recuperado de https://www.ncbi.nlm.nih.gov/pubmed/18952356.

Isbye, D. L., Rasmussen, L. S., Ringsted, C., \& Lippert, F. K. (2007). Disseminating cardiopulmonary resuscitation training by distributing 35,000 personal manikins among schoolchildren. Circulation, 116(12), 1380-1385. Recuperado de http://circ.ahajournals.org/content/116/12/1380.

Iserbyt, P., \& Byra, M. (2013). The design of instructional tools affects secondary school students' learning of Cardiopulmonary Resuscitation (CPR) in reciprocal peer learning: A randomized controlled trial. Resuscitation, 84(11), 1591-1595. Recuperado de https://www.sciencedirect.com/science/article/pii/S0300957213003468.

Iserbyt, P., Charlierb, N., \& Mols, M. (2014). Learning basic life support (BLS) with tablet PCs in reciprocal learning at school: Are videos superior to pictures? A randomized controlled trial. Resuscitation, 85(6), 809-813. Recuperado de https://www.sciencedirect.com/science/article/pii/S0300957214000501\#!.

Jones, I., Whitfield, R., Colquhoun, M., Chamberlain, D., Vetter, N., \& Newcombe, R. (2007). At what age can schoolchildren provide effective chest compressions? An observational study from the heartstart UK schools training programme. BMJ, 334, 1201 . Recuperado de https://www.bmj.com/content/334/7605/1201. 
Kanstad, S., Nilsen, K., \& Fredriksen, K. (2011). CPR knowledge and attitude to performing bystander CPR among secondary school students in Norway. Resuscitation, 82(8), 1053-1059. Recuperado de https://www.ncbi.nlm.nih.gov/pubmed/21531067.

Kragholm, K., Wissenberg, M., Mortensen, R. N., Hansen, S. M., Malta Hansen, C., Thorsteinsson, K., ... Rasmussen, B. (2017). Bystander efforts and 1-year outcomes in out-of-hospital cardiac arrest. New England Journal of Medicine, 376, 1737-1747. Recuperado de https://forskning.ku.dk/find-en-forsker/?pure=en\%2Fpublications\%2Fbystander-efforts-and1year-outcomes-in-outofhospital-cardiac-arrest(3d9a0952-29da-41b7-873fead543a3c254).html.

Long, A. F., Godfrey, M., Randall, T., Brettle, A., \& Grant, M. J. (2002). HCPRDU evaluation tool for quantitative studies. University of Leeds, UK: Nuffield Institute for Health. Recuperado de https://usir.salford.ac.uk/12969/1/Evaluation_Tool for Quantitative Research Studies.pdf.

Lorem, T., Palm, A., \& Wikbc, L. (2008). Impact of a self-instruction CPR kit on $7^{\text {th }}$ graders' and adults' skills and CPR performance. Ressuscitation, 79, 103-108. Recuperado de https://www.sciencedirect.com/science/article/pii/S0300957208005078\#!.

Lukas, R., Van Aken, H., Mölhoff, T., Weberc, T., Rammer, M., Wildd, E., \& Bohn, A. (2016). Kids save lives: a six-year longitudinal study of schoolchildren learning cardiopulmonary resuscitation: Who should do the teaching and will the effects last? Resuscitation, 101, 35-40. Recuperado de https://www.sciencedirect.com/science/article/pii/S0300957216000666\#!.

Malta Hansen, C., Zinckernagel, L., Ersbøll, A. K., Tjørnhøj-Thomsen, T., Wissenberg, M., Lippert, F. K., ... Folke F. (2017). Training in schools following 8 years of mandating legislation in Denmark: A nationwide survey. Journal of the American Heart Association, 6(3), e004128. Recuperado de https://www.ahajournals.org/doi/10.1161/JAHA.116.004128.

Meissner, T. M., Kloppe, C., Hanefeld, C. (2012). Basic life support skills of pre-primary and postcardiopulmonary resuscitation students: a longitudinal investigation. Scandinavian Journal of Trauma, Resuscitation and Emergency Medicine, 20(31), 20-31 Recuperado de https://www.ncbi.nlm.nih.gov/pubmed/22502917.

Miró, Ò., Díaz, N., Escalada, X., Pérez, F. J., \& Sánchez, M. (2012). Revisión de las iniciativas llevadas a cabo en España para implementar la enseñanza de la reanimación cardiopulmonar básica en las escuelas. Anales del Sistema Sanitario de Navarra, 35(3), 477-486. Recuperado de http://scielo.isciii.es/scielo.php?script=sci_arttext\&pid=S1137-66272012000300014.

Mpotos, N., \& Iserbyt, P. (2017). Children saving lives: Training towards CPR excellence levels in chest compression based on age and physical characteristics. Resuscitation, 121, 135-140. Recuperado de https://www.resuscitationjournal.com/article/S0300-9572(17)30678o/fulltext.

Peberdy, M. A., Callaway, C. W., Neumar, R. W., Geocadin, R. G., Zimmerman, J. L., Donnino, M., ... Kronick, S. L. (2010). "Part 9: Post-cardiac arrest care 2010 American heart association guidelines for cardiopulmonary resuscitation and emergency cardiovascular care. Circulation, 
122(18, suppl 3), S768-S786. $\quad$ Recuperado de http://circ.ahajournals.org/content/122/18 suppl_3/S768.

Perkins, G., Olasveengen, T., Maconochie, I., Soar, J., Wyllie, J., Greif, R., ... Nolan, J. (2018). European resuscitation council guidelines for resuscitation: 2017 update. Resuscitation, 123, 43-50. Recuperado de https://www.resuscitationjournal.com/article/S0300-9572(17)30776$\underline{1 / \text { fulltext. }}$

Petrić, J., Malički, M., Marković, D., \& Meštrović, J. (2013). Students' and parents' attitudes toward basic life support training in primary schools. Croatian Medical Journal, 4, 376-380. Recuperado de https://www.ncbi.nlm.nih.gov/pmc/articles/PMC3760662/.

Riddersholm, S., Kragholm, K., Mortensen, R. N., Pape, M., Hansen, C. M., Lippert, F. K., ... Rasmussen, B. (2017). Association of bystander interventions and hospital length of stay and admission to intensive care unit in out-of-hospital cardiac arrest survivors. Resuscitation, 119, 99-106. Recuperado de https://www.resuscitationjournal.com/article/S0300-9572(17)302952/pdf.

Salciccioli, J. D., Marshall, D. C., Sykes, M., Wood, A. D., Joppa, S.A., Sinha M., Lim P. B. (2018). Basic life support education in secondary schools: a cross-sectional survey in London, UK. BMJ Open, 7(1), e011436. Recuperado de https://www.ncbi.nlm.nih.gov/pubmed/28062467.

Sasson, C., Rogers, M. A., Dahl, J., \& Kellermann, A. L. (2010). Predictors of survival from out-ofhospital cardiac arrest: a systematic review and meta-analysis. Circ Cardiovasc Qual Outcomes, 3, 63-81. Recuperado de https://www.ncbi.nlm.nih.gov/pubmed/20123673.

Schroeder, D., Ecker, H., Wingen, S., Semeraro, F., \& Böttiger, B. W. (2017). "Kids Save Lives"resuscitation training for schoolchildren: Systematic review. Anestesista, 68(8), 589-597. Recuperado de https://www.ncbi.nlm.nih.gov/pubmed/28497243.

Semeraro, F., Frisoli, A., Loconsole, C., Mastronicola, N., Stroppa, F. Ristagno, G., ... Cerchiari, E. (2017). Kids (learn how to) save lives in the school with the serious game Relive. Resuscitation, 116, 27-32. Recuperado de https://www.resuscitationjournal.com/article/S03009572(17)30200-9/fulltext.

Semeraro, F., Wingen, S., Schroeder, D. C., Ecker, H., Scapigliati, A., Ristagno, G., \& Böttiger, B. W. (2016). Kids save lives implementation in Europe: A survey through the ERC Research NET. Resuscitation, 107, e7-e9. Recuperado de https://www.resuscitationjournal.com/article/S0300-9572(16)30430-0/fulltext.

SNS - Serviço Nacional de Saúde (2017). Suporte Básico de Vida. Recuperado de http://www.inem.pt/2017/05/29/suporte-basico-de-vida/.

Tanaka, H., Ong, M. E., Siddiqui, F. J., Ma, M. H., Kaneko, H., Lee, K. W., ... Shin, S. D. (2018). Modifiable factors associated with survival after out-of-hospital cardiac arrest in the pan-asian resuscitation outcomes study. Annals of Emergency Medicine, 71(5) 608-617.e15. Recuperado de https://www.ncbi.nlm.nih.gov/pubmed/28985969. 
Tavares, A., Pedro, N., \& Urbano, J. (2016). Ausência de formação em suporte básico de vida pelo cidadão: um problema de saúde pública? Qual a idade certa para iniciar? Revista Portuguesa de Saúde Pública, 34(1), 101-104. Recuperado de https://www.sciencedirect.com/science/article/pii/S0870902515000450.

Thovar, J., Castañeda, J., \& Benazco, J. (2015). Efectividad de una intervención educativa en el conocimiento de la población escolar sobre reanimación cardiopulmonar básica. Metas de Enfermería, 18(8), 63-69. Recuperado de https://eds.b.ebscohost.com/eds/pdfviewer/pdfviewer?vid=5\&sid=968fa76c-2355-4b97ae5b-bbf9d8301c9f\%40sessionmgr104.

Toner, P., Connolly M., Laverty, L., \& McGrath, P. (2007). Teaching basic life support to schoolchildren using medical students and teachers in a 'peer-training' model-results of the 'ABC for life' programme. Resuscitation, 75(1), 169-75. Recuperado de https://www.resuscitationjournal.com/article/S0300-9572(07)00431-5/fulltext.

Waalewijn, R. A., Nijpels, M. A., Tijssen, J. G., \& Koster, R. W. (2002). Prevention of deterioration of ventricular fibrillation by basic life support during out-of-hospital cardiac arrest. Resuscitation, 54(1), 31-36. Recuperado de https://www.ncbi.nlm.nih.gov/pubmed/12104106.

Writing Committee Members (2006). ACC/AHA/ESC 2006 Guidelines for management of patients with ventricular arrhythmias and the prevention of sudden cardiac death - Executive summary. European Heart Journal, 27, 2099-2140. Recuperado de https://www.escardio.org/static file/Escardio/Guidelines/publications/VASCDguidelinesVASCD-ES.pdf.

\section{COMO CITAR ESTE ARTIGO:}

Boné, M., Loureiro, M. J., Bonito, J. (2020). Suporte básico de vida na escola: o relato da evidência. Holos. 36(6), 1-21.

\section{SOBRE OS AUTORES}

\section{BONÉ}

Doutoranda do programa doutoral em educação da Universidade de Aveiro. Departamento de Expressões do Agrupamento de Escolas de Monforte. Área: educação especial. E-mail: aurorabone@ hotmail.com ORCID ID: https://orcid.org/0000-0002-4549-5313

\section{J. LOUREIRO}

Docente e orientadora de alunos do programa doutoral em educação da Universidade de Aveiro. Departamento de Educação e Psicologia da Universidade de Aveiro. Área: avaliação educacional. E-mail: mjoao@ua.pt

ORCID ID: http://orcid.org/0000-0002-5041-8689

\section{J. BONITO}

Docente do doutoramento em ciências da educação da Universidade de Évora. Orientador de alunos do doutoramento em educação da Universidade de Évora e do programa doutoral em educação da Universidade 
de Aveiro. Departamento de Pedagogia e Educação da Escola de Ciências Sociais da Universidade de Évora. Área: Didática, desenvolvimento curricular e tecnologias educativas. E-mail: jbonito@uevora.pt ORCID ID: http://orcid.org/0000-0002-5600-0363

Editor(a) Responsável: Francinaide de Lima Silva Nascimento Pareceristas Ad Hoc: ANA LÚCIA HENRIQUE E ANTÓNIO DIAS

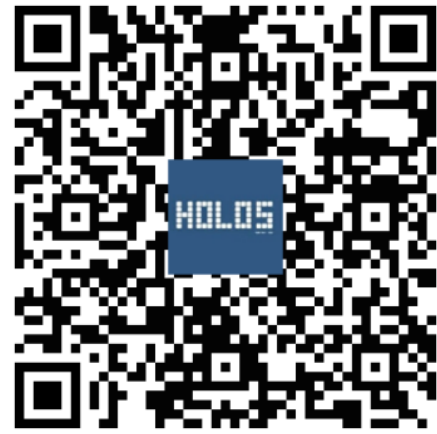

